\title{
El chocolate antes de la Gran Guerra: una perspectiva desde los sistemas abiertos*
}

\section{Chocolate before the Great War: An Open Systems perspective}

\author{
Óscar Granados \\ Profesor asociado \\ Universidad de Bogotá Jorge Tadeo Lozano, Bogotá D. C. - Colombia \\ http://orcid.org/0000-0002-4992-8972 \\ oscarm.granadose@utadeo.edu.co
}

Fecha de recepción: 12 de mayo de 2017

Fecha de aceptación: 04 de agosto de 2017

\begin{abstract}
Sugerencia de citación: Granados, Ó. M. (2017). El chocolate antes de la Gran
Guerra: una perspectiva desde los sistemas abiertos. tiempo\&economía, 4(2), 67-88, doi: http://dx.doi.org/10.21789/24222704.1222
\end{abstract}

\section{RESUMEN}

Este artículo evalúa la transformación del consumo global de chocolate en el periodo previo a la Gran Guerra (1870-1914). La investigación revela que el consumo de chocolate, más allá de una relación causal del mejoramiento en el ingreso y la búsqueda de un mejor estatus social, emergió de una interacción continua de diversos elementos como la innovación

* Este artículo es el resultado del proyecto "Banqueros, diplomáticos y bienes básicos estratégicos en América del Sur", financiado por la Universidad de Bogotá Jorge Tadeo Lozano, Bogotá, Colombia. Quiero agradecer a mi asistente Natalia Pinzón y a los participantes en los siguientes eventos: Economic and Business History Association Conference 2013, Annual Congress of the European Business History Association 2013 y Congress of the Caribbean Economic History Association 2015 donde recibí varios buenos comentarios de versiones anteriores en inglés de este documento. 
chocolatera, el emprendimiento, el comercio, la banca, la economía política y la política imperial. Por lo tanto, el consumo de chocolate funcionó como un sistema abierto.

Palabras clave: bienes básicos, globalización, imperialismo, innovación, economía política, historia económica

Códigos JEL: N40, N50, N70, N80

\section{ABSTRACT}

This article evaluates the transformation of the global consumption of chocolate in the period before the Great War (1870-1914). The research reveals that the chocolate consumption, beyond from a causal relationship between the improvement of income and the search for a better social status, emerged from a continuous interaction of different elements such as chocolate innovation, entrepreneurship, trade, banking, political economy and imperial policy. Therefore, the chocolate consumption worked as an open system.

Keywords: Commodities, Globalization, Imperialism, Innovation, Political Economy, Economic History

JEL Codes: N40, N50, N70, N80 


\section{Introducción}

La historia del chocolate está hecha de largas expediciones, exóticos sabores, llamativas innovaciones, nacientes industrias, poderosos imperios e idearios de lujo donde varias personas corrían a las vitrinas de las confiterías para sentir los aromas y sabores del chocolate. Aunque el fruto que lo originó puede ubicarse en algún punto de América Latina y el desarrollo tecnológico que posibilitó su transformación podría atribuirse a actores específicos en Europa, es necesario estudiar este proceso como el resultado de la interacción de diversas dinámicas a escala global. Así, a partir del estudio de la articulación de esas dinámicas se puede comprender cómo se logró la masificación de su consumo y cuáles han sido los diferentes significados del chocolate. Habitualmente, se ha determinado que durante la primera globalización se tuvo la posibilidad de adquirir un mayor volumen de chocolate por el mejoramiento del ingreso que trajo este periodo de ebullición económica, científica, política, social y cultural'1.

Pero, ¿la evolución del consumo de chocolate fue resultado del mejoramiento del ingreso de la clase media trabajadora? Esta pregunta se ha formulado por varios académicos y ha sido abordada desde diferentes ópticas historiográficas: la historia social, la historia de la comida, la historia del consumo, la historia natural, la historia económica y empresarial. Desde la historiografía del consumo, los trabajos de Norton $(2006 ; 2008)$ establecen que "el gusto europeo por el chocolate surgió como un accidente contingente del imperio", pero también desde una percepción sociológica como la de Bourdieu (1984), que incorpora los determinantes estéticos y las jerarquías sociales como parámetros de un consumo que generó distinción y estatus a los ojos de la sociedad. Por otro lado, Jan de Vries (1994) menciona que el deseo masivo de bienes de lujo pudo motivar a la gente a trabajar más y poder pagar sus nuevos hábitos de consumo; mientras Moss y Badenoch (2009) establecen que el chocolate empezó a asociarse con una aristocracia adicta al lujo. Wolf (1982) y Mintz (1985) argumentan que el consumo se incrementó por la estimulación energética que daba a los trabajadores para lograr mantenerse durante jornadas laborales más prolongadas.

Otros académicos han analizado la integración de los mercados del cacao y el chocolate como los trabajos de Dand (2011) y Clarence-Smith (2000) o la producción de cacao y algunas implicaciones en los periodos previos y posteriores a la Gran Guerra, también de Clarence-Smith $(1990 ; 1994 ; 1995)$, Clarence-Smith y Ruf (1996); los trabajos de Satre (2005) y Mikell (1992) que abordan el impacto social y político del cultivo de cacao en África, o los trabajos específicos de Harwick (1996), Greenhill (1996), Maiguashca (1996) y Phillips-Lewis (1996) de Venezuela, Brasil, Ecuador y Trinidad respectivamente. Aunque no lo hace directamente, el estudio de economía histórica y econometría de Weymar (1968) también da aproximaciones de la dinámica del mercado del cacao a partir de las variaciones de precios y su impacto en la demanda. Recientemente, un grupo de investigadores liderados por Johan Swinnen de la Universidad KU Leuven de Bélgica desarrollaron una serie de propuestas referentes a la economía del chocolate, y en la sección histórica se destaca una reedición de la evolución del consumo de Clarence-Smith

1 Los niveles de renta per cápita antes de iniciarse la Primera Guerra Mundial eran el reflejo de los avances industriales de las economías centrales, que dejaban rezagados a importantes imperios del siglo XIX. Mientras la renta per cápita en Estados Unidos ascendía a 377 dólares contra 244 de Gran Bretaña, 184 de Alemania y 153 de Francia, en Austria-Hungría y Rusia no superaban los 60 dólares (Kennedy, 1987).

tiempo\&economía

Vol. 4 N. ${ }^{\circ} 2$ - Julio - Diciembre de 2017 
(2016) y la transformación de algunas empresas productoras de chocolate suizas (Fromm, 2016) y belgas (Garrone, Pieters, y Swinnen, 2016).

¿Cómo se puede explicar la transformación del consumo global de chocolate durante la primera globalización si las importaciones de cacao por parte de los productores de chocolate crecieron en el periodo un promedio de $23,8 \%$ anual mientras el ingreso laboral no alcanzaba un incremento del $5 \%$ anual? El contexto de estudio, entonces, se articula en un periodo de expansión global donde se integraron la innovación tecnológica, la economía política, la estrategia imperial y la expansión de la producción de cacao. En otras palabras, la transformación del consumo global de chocolate debe ser evaluada como un sistema abierto, ya que durante el periodo de la primera globalización interactuaron varios elementos que no son separables. Primero, la innovación y la transformación industrial de las economías centrales (Alemania, Gran Bretaña, Francia, Estados Unidos) y otros países con un alto consumo de chocolate como Suiza, Bélgica y Holanda. Segundo, la fractura de la tradición del comercio del cacao liderado por el imperio español y portugués, a partir del apoyo que otros imperios dieron a sus colonias para aprovechar el incremento en la demanda de cacao que hacía parte de su estrategia de comercio internacional. Tercero, la oferta de cacao que era abastecida por países independientes como Ecuador, Brasil y Venezuela, la cual sería complementada por la producción de territorios coloniales en el Caribe, África Occidental y la península Malaya como resultado de los propósitos económicos y políticos de los imperios.

El objetivo de este documento es evaluar la evolución del consumo global de chocolate durante el periodo de 1870 a 1914 a partir de la interacción de la innovación chocolatera, el emprendimiento, la economía política, el comercio, la banca, la política imperial y la estrategia de algunos países de aprovechar sus territorios coloniales o territorios donde ejercieron influencia, para desarrollar los cultivos de cacao y mantenerse en el abastecimiento global del proceso industrial. Dicho de otra manera, el propósito es explorar nuevas formas de entender la evolución del consumo de chocolate desde la historia global y los sistemas abiertos ${ }^{2}$ articulando las diversas interacciones que facilitaron la democratización del consumo de chocolate y la transformación de las áreas cultivadas, lo que fortalece la perspectiva de Norton (2008) de que la historia del chocolate y el cacao deben entenderse como el "resultado histórico de una conjunción de factores" (p. 142). Es decir, se renuncia a una jerarquía de eventos perfectos, a la exclusiva relación causal del mejoramiento en el ingreso de la población y la búsqueda de un mejor estatus, ya que el consumo global de chocolate no fue un sistema cerrado y lineal, sino que fue un sistema influenciado por diversos aspectos externos a él, con un comportamiento dinámico de relaciones no lineales y en permanente adaptación ${ }^{3}$.

Este documento se divide de la siguiente forma: una primera sección establece la ruta global del chocolate a partir de las directrices establecidas por los empresarios y la innovación chocolatera; una segunda presenta la transformación del comercio mundial, la diplomacia y

2 Por sistema abierto se entiende aquellos sistemas que funcionan con el medio que los rodea, que interactúan con factores que no forman parte del propio sistema, que intercambian materia y energía con el mundo circundante y se vinculan con otros sistemas diferentes a ellos mismos (Meadows, 2008; Volkenstein, 1986). Es decir, el sistema de consumo de chocolate se vinculó con el medio que lo rodea y con otros sistemas diferentes al que conforma el consumo, intercambiando información, materia y energía.

3 Para una profundización de la teoría de sistemas abiertos, dinámicos y adaptativos, véanse Miller y Page (2007), Sametband (1994), Wheatley (1992). 
la estrategia de los imperios en el negocio del cacao; una tercera sección evalúa la economía política del cacao, y finalmente se presenta una conclusión.

\section{Innovación chocolatera}

Los cambios que se dieron en la importación de cacao no fueron un proceso exclusivo de la innovación de la segunda mitad del siglo XIX, pues previamente se había iniciado un acercamiento al producto. El cacao llegó a Europa por España, en el siglo XVI, en pocas cantidades a través de algunos comerciantes y órdenes religiosas que, según Norton (2008), pueden considerarse los primeros consumidores en el viejo continente. Además, llegó a manos de miembros de la aristocracia y la nobleza que lo consumió haciendo uso de las especias provenientes de las Indias Orientales y de los ornamentos que se usaban para prepararlos. No obstante, solo hasta el siglo XVIII este ingrediente empieza a aparecer en los libros de cocina europea, en los que se explica cómo hacer la bebida, la fabricación de postres, los ingredientes adecuados y la forma correcta de usarlos, la manufactura del chocolate y cómo podía ser incluido en el desayuno (Knapp, 1920; Moss y Badenoch, 2009).

Uno de los enfoques más interesantes respecto al consumo, aparte de la subsecuente asociación con sus valores nutricionales, fue la relación con el género masculino o femenino y con la clase a la que pertenecen según la presentación del producto. Para el siglo XVII, el chocolate era asociado con la masculinidad no solo porque podía ser un reemplazo para las bebidas fuertes, sino porque estaba asociado con las coffee house de la época. Para el siglo XVIII, además de mantenerse la asociación del chocolate y la aristocracia -pues era, entre otras cosas, un símbolo de sus vicios y virtudes-, se fortalece una asociación entre el chocolate y la feminidad no solo por su connotación erótica, sino por la asociación al romance. El siglo XIX, no obstante, se convierte en un momento importante hacia la democratización de este producto por cuatro procesos: el cacao empezó a crecer en nuevas regiones donde no era ni botánica ni culturalmente nativo, la mecanización de su producción, los nuevos canales de difusión y la participación de Estados Unidos como consumidor y productor del mismo (Coe y Coe, 2007). Allí, tal como había sucedido en Inglaterra, el chocolate se convirtió en un suplemento perfecto para los soldados por la posibilidad de cargarlo fácilmente y ser una fuente de energía.

En la segunda mitad del siglo XIX, las empresas que empezaron a producir chocolate y a mejorar sus técnicas se enfocaron en dos elementos. Primero, los precursores de la innovación en la industria chocolatera fueron parte esencial de un proceso tecnológico que se presentó en diferentes escenarios productivos en Europa Occidental y Estados Unidos, quienes desarroIlaron la industria, pero también condicionaron el mercado del cacao. Las estructuras empresariales fueron las que facilitaron el dinamismo del negocio del chocolate, su rápida difusión y acceso a una población más amplia, recordando que durante años había sido consumido por un grupo selecto de élites. Era una diversificación de productos que permitió nuevas mezclas, lo que dio un avance al consumo de chocolate, pasando de beber a comer un chocolate prensado y empacado.

Las innovaciones que se desarrollaron en los centros chocolateros, que se presentan en el Mapa 1 del Gráfico 1, fueron principalmente emprendimientos aislados, con excepción del caso de Suiza y Bélgica. En Suiza, Daniel Peter y Heinrich Nestlé, procedente de Frankfurt, se 
instalaron en Vevey a orillas del Lago Leman; Rodolphe Lindt, en Berna; Philippe Suchard, en Neuchâtel; Jean Tobler y Johann Sprüngli, en la región de Zúrich (Cadbury, 2010). Lo mismo sucedió con Jean Neuhaus, un emigrante suizo que se instaló en Bruselas y desarrollo una industria chocolatera que aportó en las innovaciones en los siguientes años con su empresa Neuhaus y sus descendientes. Por otro lado, Charles Neuhaus con Cote d'Or, fundada en 1883, fue otro de los desarrolladores de nuevos productos para la industria chocolatera y Eugene Callebut, que en 1850 inició sus invenciones en Bélgica, pero no sería hasta 1911 cuando su nieto Octaaf desarrolló nuevos esquemas productivos en la industria ${ }^{4}$. No obstante, otros innovadores habían participado previamente de la transformación en Suiza y Bélgica. Los desarrollos de Coenraad Van Hauten, en 1828, promovieron una primera ola de industrialización y mecanización de la producción de chocolate en Holanda y los de Antoine Menier cuando en 1816, en su farmacia a las afueras de París, empezó a hacer chocolate.

En Gran Bretaña, el desarrollo de la industria se concentró en el movimiento fundado por George Fox, la Sociedad Religiosa de los Amigos, de donde los mayores chocolateros británicos -Joseph Rowntree, Francis Fry y Richard Cadbury- desarrollaron centros industriales en York, Bristol y Birmingham respectivamente. Otros empresarios le imprimieron dinamismo al negocio chocolatero, como la familia Stollwerck en Colonia, Cornellius Hollander en Ámsterdam, Taylor Brothers, Dunn \& Hewett en Londres o John Mackintosh en Halifax, quien entró al negocio del chocolate en 1912 después de su éxito en el negocio confitero (Fitzgerald, 2000). Finalmente, Milton Hershey con el apoyo de J. M. Lehmann, quien impulsaría el avance de la innovación en Estados Unidos (D’Antonio, 2006). Tal como se observa en el Mapa 1 de la Gráfico 1, se construyó una aglomeración de innovaciones y factorías de chocolate en Europa Occidental que aprovecharon el acceso a la materia prima: una ruta del cacao como presenta el Mapa 2. Sumado a esto, se estableció una estrategia de mercadeo que se apalancaba en las nuevas técnicas de publicidad, donde algunas resaltaban el lujo y otras las diferentes bondades del chocolate (Fraser, 1981).

El incremento en la producción requirió de un abastecimiento constante de materia prima que permitiera atender una demanda creciente. Las ventas de empresas como Cadbury pasaron de 117.505 libras esterlinas en 1880 a 706.191 en 1895, un crecimiento promedio anual de $33,4 \%$, así como un crecimiento en la fuerza de trabajo de 800 a 2600 empleados entre 1893 y 1899 (D’Antonio, 2006). Es decir, la nueva industrialización de las fábricas chocolateras en Europa, y posteriormente en Estados Unidos, mostró una ampliación de la producción intensificando el uso de materia prima colonial.

Esto permite argumentar el segundo elemento: la publicidad resaltó la importancia de la familia en la época y ya no mostró al chocolate como un lujo de la aristocracia, sino como un alimento nutritivo y saludable que brindan las madres a sus hijos para cuidar de ellos. Esto sumado a la presentación del chocolate en bloques con pastillas y el desarrollo de empaques, que abrió nuevas posibilidades para la difusión del producto y la adquisición de nuevos significados y usos sociales. No obstante, al analizar de cerca la historia del consumo del chocolate es importante notar, como se dijo anteriormente, que existe una disparidad en la forma en la que el consumo de este producto se masificó, no solo en términos de significados y usos, sino también de acceso.

4 Para una interpretación de las empresas chocolateras belgas, véanse Garrone, Pieters, y Swinnen (2016) 
Gráfico 1. La ruta global del chocolate

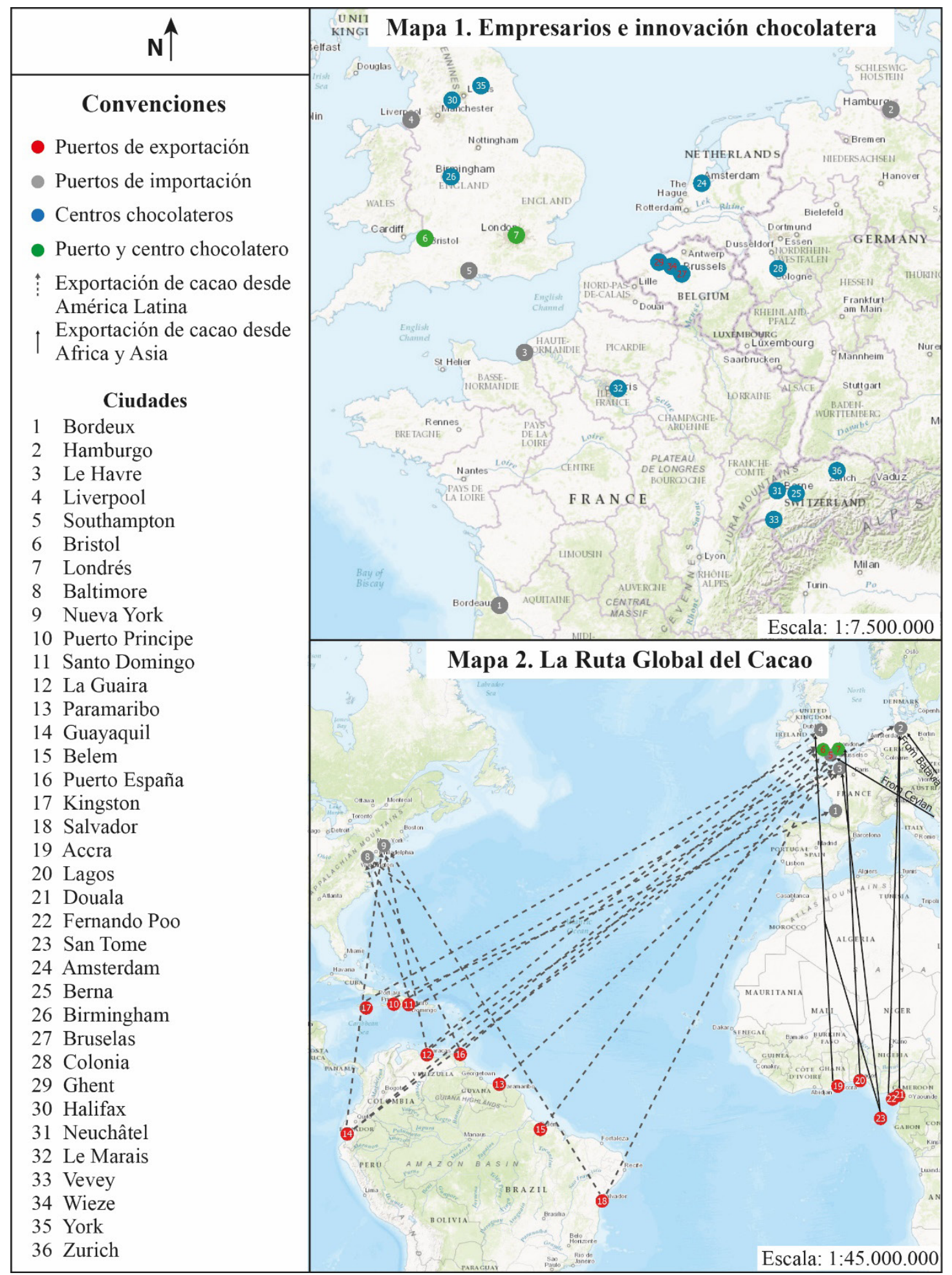

Fuente: elaboración propia. 
Esta multiplicación de las formas y empaques del chocolate e el siglo XIX fueron asociadas con la maternidad, la domesticidad y el amor romántico -pero solo para las clases medias y altas-. La discusión se volvió decididamente paternalista cuando se dirigieron a las clases trabajadoras. El chocolate sólido era para mujeres y niños de clase media y alta, pero para las familias de clase trabajadora, la cocoa se convirtió en el equivalente de una sopa caliente, nutritiva y barata. (Moss y Badenoch, 2009, p. 72)

Así, empresas como Cadbury empezaron a publicitar al chocolate como un "combustible" para la clase trabajadora. Esto resulta llamativo no solo para los obreros, sino también para los dueños de las empresas, pues ven en la promoción del consumo de chocolate una posibilidad para que sus trabajadores sean productivos, sin necesariamente mejorar sus condiciones laborales. No obstante, aquí es cuando se puede vislumbrar lo que Moss y Badenoch llaman una ironía en el consumo del producto, dado que mientras los niños y mujeres burguesas eran los consumidores más visibles, la expansión de la industria y su creciente mecanización convirtieron a niños y mujeres en una de sus fuerzas de trabajo más importantes (Moss y Badenoch, 2009, p. 72). De hecho, la división de labores que estableció esto permitió que se garantizara socialmente la calidad del producto, pues mientras los hombres hacían los chocolates, las mujeres cuidaban los detalles del empaque.

Como lo indica la Tabla 1, las importaciones de aproximadamente 36.000 toneladas en 1885 pasaron a más de 237.000 toneladas en 1914, un crecimiento promedio anual de $23,8 \%$ para los principales productores de chocolate. Entre los niveles de crecimiento anual promedio en la importación de cacao durante el periodo 1885 a 1914, se destacaron Holanda con 106,9\%, Alemania con 58,62\% y Estados Unidos con 50,35\%. Sin embargo, Estados Unidos se convirtió rápidamente en el mayor consumidor de cacao, pasando de unas importaciones en 1870 de 418.000 dólares a más del triple para 1885 por valor de 1.33 millones de dólares y para 1911 de 14.55 millones de dólares ${ }^{5}$.

Tabla 1. Importaciones de cacao de principales consumidores, 1885-1914

\begin{tabular}{cccccccc}
\hline País & 1885 & 1895 & Var. \% & 1905 & Var. \% & 1914 & Var. \% \\
\hline Estados Unidos & 5 & 13 & 160,0 & 32 & 146,2 & 78 & 143,8 \\
Alemania & 3 & 10 & 233,3 & 30 & 200,0 & 54 & 80,0 \\
Holanda & 1 & 3 & 200,0 & 11 & 266,7 & 32 & 190,9 \\
Gran Bretaña & 7 & 14 & 100,0 & 20 & 42,9 & 30 & 50,0 \\
Francia & 12 & 15 & 25,0 & 22 & 46,7 & 26 & 18,2 \\
Suiza & 1 & 2 & 100,0 & 5 & 150,0 & 10 & 100,0 \\
España & 7 & 7 & 0 & 6 & $-14,3$ & 7 & 16,7 \\
\hline
\end{tabular}

Nota: en miles de toneladas métricas.

Fuente: elaboración propia con información de Chicago Board of Trade Records (CBOT), Organizational Records, Annual Reports y Clarence-Smith (2000).

5 CBOT, Annual Reports 1911, Series I, Organizational Records, Box 87; CBOT, Annual Reports 1911, Series I, Organizational Records, Box 92. 
Para 1900, el mercado de chocolate en Gran Bretaña produjo 69.000 toneladas métricas de confitería de chocolate y para 1913 pasó a 114.000 toneladas métricas, por valor de 10.1 y 19.7 millones de libras esterlinas respectivamente (Fitzgerald, 2000). Es decir, una tonelada de chocolate tenía un valor de 146.38 libras esterlinas en 1900 contra 170.18 libras esterlinas en 1913. Como resultado, el margen entre la materia prima y el producto final para 1913, con un precio promedio de las variedades de cacao entre 31.51 y 51.89 libras esterlinas que se transaban en ese año en el puerto de Le Havre, sería de aproximadamente 118.29 y 138.67 libras esterlinas, un valor añadido que superaba el $228 \%$; una razón que hacía del chocolate un negocio lo suficientemente rentable para utilizar todos los mecanismos necesarios y aprovechar la bonanza del incremento en el consumo en Europa y Estados Unidos y de las importaciones crecientes de las élites en la periferia (Hernández, 2008; Norton, 2006).

\section{Diplomacia del chocolate}

El segundo elemento que se evalúa es la dinámica de la política imperial, la cual buscó afianzar el acceso a los recursos que se demandaban cada vez más en las economías centrales como parte de la competencia industrial mundial ${ }^{6}$. Esto benefició a los productores de chocolate al integrar la consolidación terrateniente y las nuevas tierras cultivables en África y en menor medida en el Caribe y Suramérica. A su vez, las políticas de comercio internacional favorecieron el avance de los imperios y redujeron el impacto en su balanza comercial con otras regiones, beneficiando a los empresarios con costos locales para el uso de bienes básicos y ampliando la compra de sus productos manufacturados por parte de la periferia?.

Ante una mayor demanda por parte de las economías centrales, además de otros países como Suiza, Holanda y Bélgica, las casas mercantiles estaban interesadas en que los avances de los cultivos africanos les abrieran posibilidades para comerciar bienes europeos y también para vender el cacao a los compradores británicos en los diferentes puertos (Green y Hymer, 1966, p. 304). La red del comercio mundial aprovechó los puertos de Londres, Southampton, Liverpool y Bristol, que se convirtieron en los centros de acopio del cacao en Gran Bretaña; Bordeaux y Le Havre en Francia; Hamburgo en Alemania; Baltimore y Nueva York en Estados Unidos. El comercio no se dio exclusivamente en cabeza de compañías imperiales como en la época de los imperios de puertas cerradas, sino bajo la interacción de empresas mercantiles de diferentes latitudes, que se convirtieron en el eslabón que integró al cacao con el chocolate. La misión Basel y su subsidiaria mercantil, la United Trading Company (UTC) con la German West Africa Trading Company, African Association, Swanzy y Millers serían parte de la consolidación de las exportaciones ghanesas de cacao y su vinculación al mercado suizo (Guex, 1998; Southall, 1978). Mientras, John Holt \& Co. y Ambas Bay en Liverpool se convirtieron en los mayores compradores de los agricultores de Camerún y los territorios de Fernando Poo; igualmente, las casas mercantiles

6 El Departamento Imperial de Agricultura para las Indias Occidentales se convirtió en pieza fundamental para avanzar en la consolidación de la producción de cacao en el Caribe y sus resultados investigativos fueron implementados y modificados en África y Ceylán, este último afectado también por la enfermedad del cacao (Bulletin of the Imperial Institute, 1906, pp. 351-353; Bulletin of the Imperial Institute, 1908, p. 205).

7 Una perspectiva, desde las colonias, del comercio entre las colonias británicas y la metrópoli puede verse en Greaves (1954, pp. 4-5).

tiempo\&economía

Vol. 4 N. ${ }^{\circ} 2$ - Julio - Diciembre de 2017 
de Hamburgo, Woermann y Jantzen \& Thormählen facilitaron créditos a los agricultores ejerciendo funciones de bancos mercantiles (Clarence-Smith, 1995; Pedler, 1974).

Detrás de estas empresas también existieron casas mercantiles y especuladores que participaron en la construcción de precios, aprovechando el incremento de la demanda por parte de los productores de chocolate y alejando a los agricultores de la intermediación. Con excepción de las élites en Ecuador y San Tomé, pues aprovecharon en mayor medida el comercio de cacao y las grandes extensiones de tierra cultivadas, emergiendo como una burguesía que se convirtió en una clase nacional que disfrutó de una vida en París, para el caso de las familias ecuatorianas, y Lisboa, para los plantadores de San Tomé, como José Constantino Días. También, los nuevos terratenientes alemanes en Camerún, como Max Esser, se identificaron con las plantaciones corporativas y con la vinculación en los asuntos públicos de su país (Clarence-Smith, 1995; Guerrero, 1980; Weinman, 1970).

Con la producción africana de Ghana y Camerún se inició una nueva construcción de precios como lo presenta el Gráfico 2, donde las exportaciones de los primeros años del siglo XX se conectaron fácilmente con la intermediación y los precios en los puertos. Ante esta interacción comercial, los precios del cacao oscilaban para 1913 en el puerto de Le Havre, según su origen, así: el cacao venezolano entre 30.95 y 79.37 libras esterlinas por tonelada métrica; el cacao de Ceylán entre 33.73 y 41.77; los granos de Pará y Bahía en Brasil, Guayaquil en Ecuador, San

Gráfico 2. Precio del cacao de Ghana (1893-1914) y Nigeria (1901-1914)

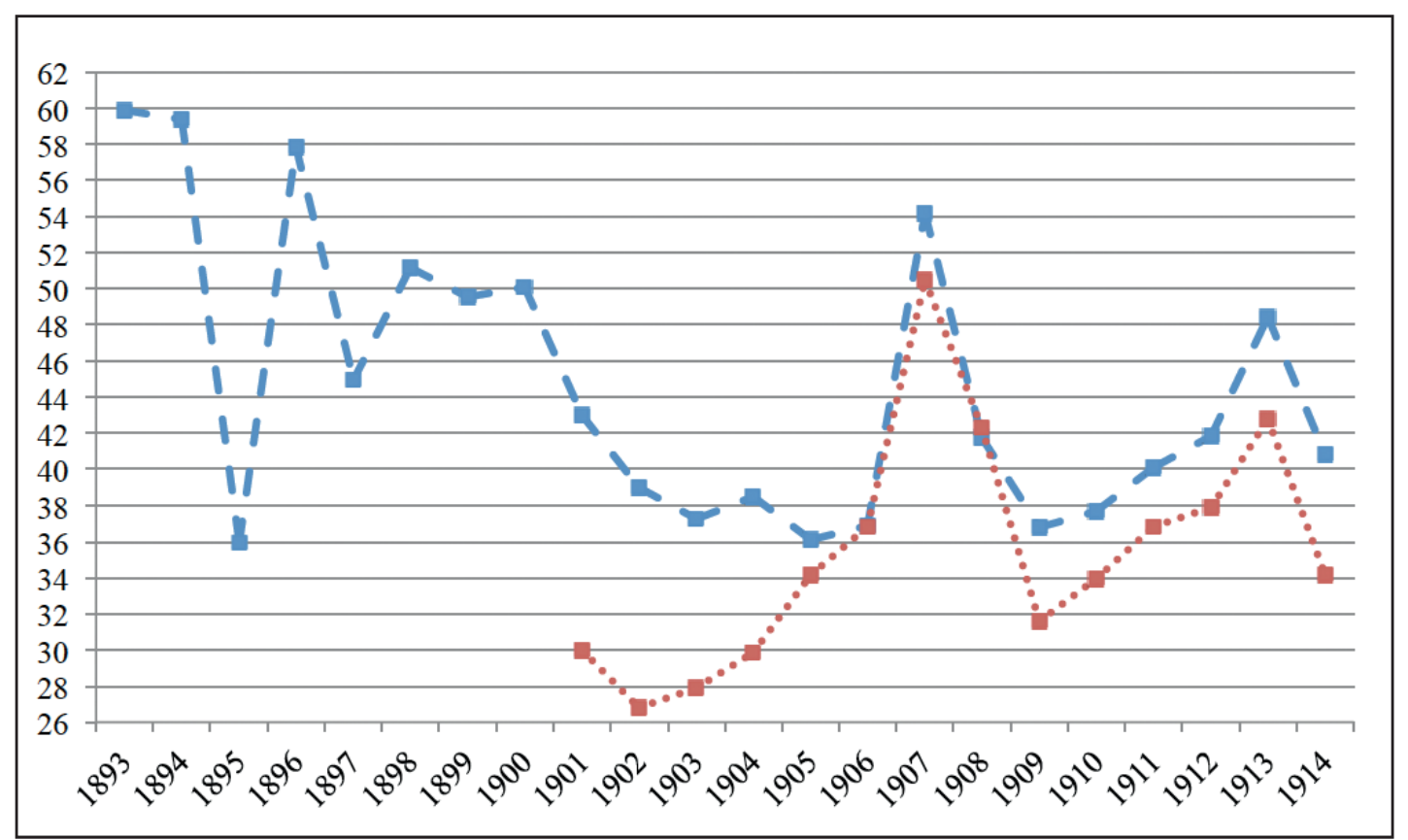

Nota: Los precios están expresados en libras esterlinas por tonelada métrica. El precio es construido a partir del volumen en libras exportado por el valor total de Ghana y la serie corta del sur de Nigeria, multiplicado por la cantidad de libras (220.46) de una carga de 100 kilos, donde se requieren de 10 cargas para una tonelada métrica.

Fuente: Elaboración propia con información de Colonial Reports Annual. Gold Coast, \#603, \#654, \#688, \#725, \#806; Colonial Reports Annual. Southern Nigeria, \#433, \#512, \#583, 825; Colonial Reports Annual. Nigeria, \#920. 
Tomé y Ghana en África se mantenían entre 29.76 y 34.52 libras esterlinas por tonelada métrica de acuerdo con el trabajo de Tosta Filho ${ }^{8}$. Es decir, los precios del cacao africano fueron más competitivos porque se acercaban a los precios de los cacaos estándar de Ghana y aquellos de una mejor calidad, como el cacao brasilero, pero con la salvedad de que tenía beneficios tributarios por ser de origen colonial y, adicionalmente, su cercanía con los centros de producción de chocolate.

Por otro lado, los capitalistas religiosos británicos distribuían sus importaciones de dos cen-

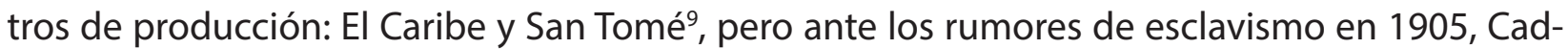
bury, Fry, Rowntree y sus socios alemanes Stollwerck presionaron para que se revisara si los portugueses aún manejaban esquemas de esclavitud, abolida desde 1875, en las plantaciones de cacao y si esto era lo que hacía más competitivo su producto (Cadbury, 2010; Clarence-Smith, 1990; Clarence-Smith, 1994). Portugal accedió ante la presión británica y germánica en 1909, pero esos esquemas esclavistas con una pequeña variación se trasladaron a las nuevas plantaciones en Ghana, Nigeria y Camerún, promocionadas por la diplomacia imperial para aprovechar la alta demanda de cacao. Años después, Cadbury y Fry iniciaron sus importaciones de las nuevas y productivas plantaciones de Ghana y para 1914 el 49,4\% del cacao usado por la industria británica era de sus posesiones coloniales (Knapp, 1920; Ross, 2014; Southall, 1978).

Igualmente, el negocio cacaotero contó con una importante inversión europea y estadounidense que permitió el desarrollo de una actividad que por sus características de cultivo requería de capital sin retorno en los primeros años. El incremento en la producción de cacao de República Dominicana y Camerún se benefició con la inversión alemana y la conexión con las casas mercantiles que permitieron la exportación del cacao. Los comerciantes británicos, alemanes y franceses ampliaron sus oportunidades de negocio en el Caribe, Suramérica, África y Asia, porque consideraban que serían respaldados por sus propios gobiernos, ya que sus actividades servían a los intereses comerciales nacionales y a la adquisición de tierras, como lo hizo Alemania en algunas regiones del Caribe o los ingleses y franceses en la región de la Costa de Oro en África (Callcott, 1942; Plummer, 1981). Es decir, los grandes productores británicos y alemanes utilizaron su influencia para presionar a sus cancillerías con el fin de que la diplomacia fuera parte de su estrategia corporativa.

La ruta del cacao tuvo como característica principal el cambio de los mayores productores de países independientes, como Brasil, Ecuador y Venezuela a territorios coloniales en África y Asia, así como la continuidad de los territorios de las Indias Occidentales y en países independientes en el Caribe asechados por los intereses estadounidenses y alemanes, como Cuba, República Dominicana y Haití. De esta forma, la producción de 1885 estaba distribuida entre países independientes y territorios coloniales en $66,2 \%$ y $33,8 \%$ respectivamente. Antes de la Gran Guerra, Ghana se consolidó como el mayor productor de cacao, pasando de una exportación de 30 libras por valor de 4 esterlinas en 1891 a 12.927 toneladas en 1908 por valor de 540.821 esterlinas y para 1914 ascendía a 53.750 toneladas por valor de 2.19 millones

8 El trabajo de Tosta Filho utiliza los precios de carga de 100 kilos en francos franceses, en este documento son convertidos a libras esterlinas utilizando un tipo de cambio de 25.2 francos franceses por libra esterlina, multiplicado por 10 para establecer como unidad la tonelada métrica. Véase Tosta Filho (1957, p. 3).

9 Para 1900, se importaba de San Tomé casi el 45\% de la demanda británica.

tiempo\&economía

Vol. 4 N. ${ }^{\circ} 2$ - Julio - Diciembre de 2017 
de esterlinas ${ }^{10}$; mientras Ecuador se consolidó como el mayor productor independiente. Los puertos de exportación aumentaron su población, pero no necesariamente su riqueza: Guayaquil pasó en 1880 de 36.000 habitantes a 89.771 en 1920, un crecimiento promedio anual de 3,73\%; mientras Accra pasó de 19.582 habitantes en 1911 a 42.803 en 1921, un crecimiento promedio anual de 11,86\% (Brand, 1972; Guerrero, 1980; Tudhope, 1909).

Asimismo, la inversión ferroviaria facilitó la integración de los cultivos con los puertos de Acraa y Seccondee, en el caso de Ghana, y los ferrocarriles y propuestas en Ecuador por parte de Speyer Brothers; Baring y sus socios en Estados Unidos, Archer Harman y Edward Morely, así como de WR Grace \& Co. y sus socios ${ }^{11}$. También, fue parte del respaldo dado por el gobierno imperial alemán a los propósitos privados en África occidental, que le permitieron proclamar su política colonial a pesar que varios desarrollos ferroviarios en las colonias alemanas fueron respaldadas por inversionistas británicos, así como la promoción de los cultivos de cacao en Camerún ${ }^{12}$. En otras palabras, la inversión en infraestructura y las actividades comerciales fueron necesarias para poder integrar la materia prima al consumo creciente de chocolate, donde la política imperial y la diplomacia direccionaron la integración de las zonas de producción cacaotera con la fabricación de chocolate.

Como lo indica la Tabla 2, hubo un cambio drástico en el mapa de producción cacaotera en un periodo muy corto, donde los países independientes pasaron a tener menos del $50 \%$ de la producción de cacao en 1914 y los territorios coloniales pasaron al 52,8\% de la exportación global. Algunos productores de chocolate empezaron a enfocarse más en la materia prima procedente de África como los británicos y los españoles, mientras otros perdieron influencia en el mercado como los holandeses ${ }^{13}$. Esta transformación llevó a los países independientes y los territorios coloniales a buscar el apoyo de sus gobiernos, promoviendo la diplomacia del chocolate durante un periodo esencial para los negocios internacionales y buscando esquemas que los beneficiaran o, por lo menos, les permitieran mantenerse en el negocio.

10 Los datos originales están en libras. Colonial Reports Annual. Gold Coast, \#806; Colonial Reports Annual. Gold Coast, \#809.

11 Una evaluación de las vías del comercio en el África tropical. Véase Barker (1925). Para el caso de Ecuador, véase: Reporte de Archer Harman en Nueva York "Guayaquil \& Quito Railway Ecuador Report" Londres 1897 para la gerencia de la suscripción de la sindicación de recursos, Baring Archive, House Correspondence, Series HC4, 4.4.38; Carta de Edward Eyre a Grace Brothers en Londres "Ecuadorian Business" Nueva York 4 de abril de 1902, WR Grace \&Co. Collection (WRGC), Serie III 5: Letterbooks, Box 74, \#17, Folio 193-195.

12 El respaldo dado por el imperio alemán a los propósitos terratenientes de Herr Liideritz en África Occidental y su respectiva comunicación al Imperio Británico el 24 de abril de 1884, se reconoce como el inicio de la política colonial alemana, véase: (Crabtree, 1914, p. 7). A pesar de este respaldo, los inversionistas alemanes no tenían experiencia en financiar actividades productivas en regiones apartadas y fuera de Europa (Bulletin of the Imperial Institute, 1906, p. 68). Además, la influencia permanente de Bismarck en los bancos privados para no buscar inversiones fuera de las fronteras europeas, contra los avances del banco por acciones Diskonto-Gesellschaft, que se vinculó con la financiación de emprendimientos coloniales y uno de los bancos más activos en los mercados periféricos desde su fundación. Véase (Henderson W. O., 1945, p. 56; Stern, 1977).

13 Las enfermedades del cacao de Surinam empezaron a afectar la competitividad de Holanda en el negocio del cacao (Bulletin of the Imperial Institute, Great Britain: Commonwealth Institute, 1906, p. 348). 
Tabla 2. Exportación de cacao por grupo de países independientes y territorios coloniales

\begin{tabular}{|c|c|c|c|c|c|c|c|c|c|}
\hline País/Territorio & 1885 & $\begin{array}{c}\text { Part. } \\
1885 \\
\%\end{array}$ & 1895 & Var. \% & 1905 & Var. \% & 1914 & Var. \% & $\begin{array}{c}\text { Part. } \\
1914 \%\end{array}$ \\
\hline Independientes ${ }^{\mathrm{a}}$ & 26,9 & 67,9 & 38,2 & 42 & 71,5 & 87,2 & 131,9 & 84,5 & 47,1 \\
\hline Britanico $^{b}$ & 9,7 & 23,8 & 19,9 & 105,2 & 38,4 & 92,9 & 100,2 & 160,9 & 35,8 \\
\hline Frances $^{c}$ & 0,6 & 1,6 & 0,8 & 33,3 & 1,5 & 87,5 & 2,3 & 53,3 & 0,8 \\
\hline Alemán ${ }^{d}$ & 0 & 0,0 & 0,1 & N.A. & 1,4 & 1300 & 4,4 & 214,3 & 1,6 \\
\hline Español $^{\mathrm{e}}$ & 0,7 & 1,9 & 1,9 & 171,4 & 1,9 & 0 & 4,8 & 152,6 & 1,7 \\
\hline Portugués $^{f}$ & 1,2 & 2,9 & 7,0 & 483,3 & 25,7 & 267,2 & 32,1 & 24,9 & 11,5 \\
\hline Holandés ${ }^{g}$ & 1,4 & 3,6 & 5,3 & 278,6 & 3,6 & $-32,1$ & 3,5 & $-2,7$ & 1,3 \\
\hline
\end{tabular}

Nota: en miles de toneladas.

a Los países independientes son: Ecuador, Venezuela, Brasil, República Dominicana, Haití y Cuba.

Este último en las cifras de 1905 y 1914.

b Hace referencia a Jamaica, Granada, Dominica, San Vicente, Trinidad y Tobago, Sri Lanka, Nigeria y Ghana.

c Son los territorios de Guadalupe y Martinica.

¿ Son los territorios de Camerún.

e Son los territorios de Cuba hasta 1898 y Guinea Ecuatorial.

f Son los territorios de San Tomé.

g Son los territorios de Surinam y las Indias orientales holandesas (Indonesia).

Fuente: elaboración propia con información de Clarence-Smith (2000).

\section{Economía política del cacao}

La economía política del cacao se definió a partir de tres aspectos: la promoción de exportaciones, la política fiscal y el endeudamiento público. Al iniciar el siglo XIX, las exportaciones mundiales de cacao ascendían a 11.000 toneladas donde el $41 \%$ correspondía a Venezuela, $22 \%$ a Ecuador y $21 \%$ a Brasil; es decir, el $84 \%$ de las exportaciones se concentraban en tres productores. Para 1850, la expansión se trasladó al Caribe, donde se ampliaron las plantaciones en Jamaica, Santa Lucía, Granada y Trinidad; este último se integró al grupo de grandes productores a partir de la política real de parcelas para los cultivos de cacao con una participación en las exportaciones mundiales de 11,2\%, mientras Venezuela perdía su liderazgo y pasaba a tener el 21,8\%, superado por Ecuador y Brasil con el 32,8\% y 26,7\% respectivamente (Cadbury, 2010; Phillips-Lewis, 1996). A esto se sumaban las producciones de Cuba, República Dominicana, Haití, Granada y Surinam, además de pequeñas producciones en San Tomé e Indonesia que no superaban el $0,1 \%$ para finales del siglo XIX. Colombia producía aproximadamente 5.000 toneladas por año, pero su exportación no superaba el $5 \%$ debido a que el 
chocolate se fortalecía como parte de la dieta interna (Martínez, 2000), su modelo de producción era de pequeñas parcelas y tenía dificultades en la consecución de capital ${ }^{14}$.

El éxito de los tres principales productores durante el siglo XIX fue el resultado de: primero, la calidad y el aroma del cacao, que fueron fundamentales en la producción del chocolate destinado a un grupo selecto de consumidores. Segundo, el incremento de su producción en regiones como el valle del río Tuy en Venezuela y el valle del río Guayas en Ecuador a través de una constante siembra de árboles que venía desde el siglo XVII en Venezuela y Ecuador ${ }^{15}$. Esto mismo sucedió con la expansión productora en el sur del Estado de Bahía, en Brasil, que reemplazó los márgenes de la selva amazónica brasilera en la región de Pará como la región con mayor producción. Tercero, la producción se integró a una eficiente conexión (véase Mapa 2) de los puertos de exportación de La Guaira, Guayaquil, Belem y Salvador respectivamente, que permitieron mantener el tradicional abastecimiento al mercado de México ${ }^{16}$ y posteriormente el de la economías centrales ${ }^{17}$. Para 1885 , las exportaciones se elevaron a 40.689 toneladas donde América Latina participaba con el 95,7\% y diez años después participaba con el 86,5\% de la exportación de 74.185 toneladas. Para 1905, las exportaciones fueron de 145.602 toneladas, donde la producción africana y asiática, en su totalidad de territorios coloniales, llegó a 24,3\% y 2,9\% respectivamente. En 1914, las exportaciones se elevaron a 279.867 toneladas, donde el hemisferio occidental tenía el 62,3\% (véase Tabla 2).

El segundo aspecto de la economía política fue el cambio de los esquemas tributarios a la importación de cacao. En Gran Bretaña se mantuvo una tasa de 9.31 libras esterlinas por tonelada métrica entre 1898 y 1913, mientras Alemania la redujo de 17.16 a 9.80 libras esterlinas durante el mismo periodo. Francia diferenció el origen de la materia prima: al cacao extranjero le imponía una tasa de importación de 40.68 libras esterlinas por tonelada contra 20.59

14 Algunos autores establecen la influencia de la elites de Popayán en el cultivo del cacao durante la colonia en los municipios de Tuluá, Palmira, Puerto Tejada, Neiva y Garzón como una de las regiones más importantes en el cultivo, pero opacado por la explotación de oro, véanse Krug y Quartey-Papafio (1965, pp. 18-21); Marzahl (1978); Bushnell (1993). No obstante, el esquema de aprovechamiento a gran escala de las riquezas del Amazonas se trató de revivir con el propósito de los hermanos Reyes en la región, así como de la Compañía del Caquetá para estructurar grandes plantaciones en la Amazonía, véase Estatutos de la Compañía del Caquetá $(1887$, p. 7) y Reyes (1902). Mientras la producción en menor escala fue la que mejor se desarrolló a pesar de las dificultades en la consecución de capital, véase Informe del Presidente del Estado de Santander a la Asamblea Lejislativa en sus sesiones de 1873 (1873, p. 10).

15 La región cacaotera en Venezuela pasó de 433.350 árboles en 1684 a 5.118 .821 árboles en 1744, lo que llamó la atención de mercaderes vascos e inversionistas creando la Compañía Guipuzcoana (Compañía Caracas) que monopolizó la comercialización del cacao de la región y redujo la participación de los holandeses, así como el contrabando de cacao (Ferry, 1980). Aunque la región del Valle del Tuy participaba con 1.380.885 frente a 1.478.450 de la región de Barlovento, para el año de 1768 Barlovento se convertía en la mayor zona de producción con 2.447 .800 árboles contra los 1.716 .222 de la tradicional región del Tuy, sin dejar de destacar la región de la península de Paria que también avanzó en la producción de cacao (Harwich, 1996; Tamaro, 1988).

16 El Mercado de Veracruz y en especial la demanda de la Ciudad de México por el cacao de Guayaquil y Caracas fue una evolución progresiva ante la caída en la producción en Mesoamérica, que no solo sirvió como producto alimenticio, sino como medio de pago en México (del Valle Pavón, 2010, pp. 183-185). Lo que posteriormente facilitó el ingreso de estos dos productores durante el siglo XVIII, su consolidación en los años siguientes y la posterior decadencia de la producción de la Provincia de Caracas (Pinero, 1987).

17 En 1880, Francia consumía 10.767 toneladas contra 8.471 de España y lo superaba como el mayor consumidor mundial de cacao. 
libras esterlinas al cacao de origen colonial, que para 1914 se había eliminado (Girault, 1916). Mientras Holanda, Bélgica, Estados Unidos y Suiza no impusieron ningún arancel o era muy pequeño, como el impuesto suizo de 49 peniques por tonelada métrica, mientras Estados Unidos ya había suspendió cualquier impuesto desde $1886^{18}$. Sin embargo, esto fue diferente en el comercio internacional del chocolate, donde el proteccionismo entre los grandes productores como Estados Unidos, Gran Bretaña, Alemania, Francia, Suiza, Bélgica y España estableció aranceles a la importación de chocolates. Aquí, el esquema del libre mercado anterior a la Gran Guerra no se presentó y la diplomacia solo se usó en el comercio de la materia prima.

Los cambios tributarios y las tarifas preferenciales de las metrópolis beneficiaron las exportaciones y dieron sostenibilidad a la actividad agrícola colonial (Blanshard, 1947). A esto se sumó la imposición de impuestos a las exportaciones en Ecuador, República Dominicana, Brasil y San Tomé que redujeron su competitividad al incrementar los costos de exportación. Como resultado de esta decisión en los países independientes, los grandes hacendados del cacao buscaron cambiar los esquemas fiscales que perjudicaban sus utilidades. En el caso de los pequeños productores, se abandonó y descuido el cultivo del cacao, lo que generó varias enfermedades en los árboles de la región caribeña y suramericana y, además, profundizó la crisis ante una variedad más resistente en África.

Esta fue una de las razones para la caída de la producción de Trinidad y Tobago, que sería reactivada exclusivamente con los subsidios gubernamentales, ya que varios cultivos empezaron a operar a pérdida y, también, en Granada y Santa Lucía (Blanshard, 1947). Era una necesidad que el mismo Parlamento Británico quería solventar desde años atrás para reducir el excesivo gasto que tenían colonias como Trinidad, que no lograban fortalecerse nuevamente en la producción de $\mathrm{cacao}^{19}$. En contraste con el incremento en la productividad africana por la resistencia de los árboles de cacao, los territorios de las Indias Occidentales estaban reduciendo su competitividad en el negocio ante los problemas de infraestructura, la insatisfacción de los servicios de salud y el impacto de las plagas que afectaron el avance de los cultivos en el Caribe británico ${ }^{20}$.

Los grandes terratenientes en América lograron diversificar sus actividades, pero la dinámica del cultivo para los pequeños productores les dificultó su salida del negocio por la cantidad de recursos invertidos inicialmente en los cultivos. Ecuador, el mayor exportador mundial durante el periodo 1870-1914, cultivó 257.221 árboles para el año 1885; mientras para 1914 cultivó 971.678 árboles, lo que hizo que familias como los Aspiazu, los Seminario, los Morla, los Puga, los Caamaño y los Durán Ballén tuvieran propiedades que superaban el millón de árboles ${ }^{21}$, convirtiéndolos en el núcleo del negocio exportador de Ecuador. Un negocio que

18 Los datos originales (Clarence-Smith, 2000) son presentados en Marcos alemanes por cada 100 kilos. Se hace la conversión de monedas de una libra esterlina por 20.4 marcos alemanes y se utiliza la tonelada métrica como unidad de medida (mil kilogramos).

19 The Parliamentary Debates (1884, p. 263); Pathmanathan Umaharan, en discusión con el autor, 6 de noviembre de 2015.

20 The Parliamentary Debates (1890, p. 886).

21 Varias de las familias ecuatorianas establecieron sus empresas matrices en el exterior, tanto en Hamburgo como Londres y algunos con representaciones comerciales en Londres, Hamburgo, Berlín, París y Nueva York (Chiriloga, 1980); Xavier Elizarde Romero, en discusión con el autor, 9 de noviembre de 2012.

tiempo\&economía

Vol. 4 N. ${ }^{\circ} 2$ - Julio - Diciembre de 2017

p. 81

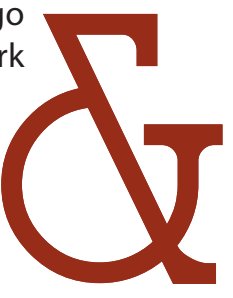


aportó al crecimiento económico, en la medida en que amplió su esquema productivo y de inversión hacia otros sectores económicos, pero nunca hacia la producción de chocolate para el caso ecuatoriano. Entre tanto, otros países que contaban con extensas áreas cultivadas no lograron trasladar su acumulación a las regiones de origen debido a que los inversionistas eran habitualmente extranjeros, con excepción de algunos casos en Venezuela y Brasil que transformaron el capital del cacao en capital financiero para abandonar el negocio o para diversificar su patrimonio.

En Ecuador, las élites se ampliaron hacia el sector bancario con participaciones en los bancos Internacional, Territorial, Comercial y Agrícola, fundado en 1895, de Ecuador y muy tímidamente al sector industrial y comercial (Henderson P., 1997; Miño, 2008). Con excepción de la Familia Aspiazu que desarrolló participaciones en el Banco Comercial y Agrícola, el Banco de Ecuador y las compañías comerciales exportadoras e industriales de una incipiente economía en transformación, frente a una diversificación estrictamente financiera de las familias Morla, Seminario y Sánchez Bruno, que paulatinamente se alejaron del mercado cacaotero ante las dificultades de la política monetaria, la especulación, la volatilidad del precio, la estructura de cultivo mundial y el traslado de Londres a Nueva York como el principal puerto de importación de cacao (Close, 1959; Weiss, 1970).

El tercer aspecto de la economía política fue el poco aprovechamiento que las regiones cacaoteras hicieron de la acumulación de capital y su transformación en capital financiero. Esto llevó a que varios países y regiones que aún no contaban con una capacidad fiscal más allá de los impuestos al comercio internacional, tuvieran que acudir a los empréstitos internacionales. Los países incrementaron su nivel de endeudamiento público, la relación de la deuda extranjera al comercio exterior era de 5,2\% para 1913, y se convirtió en un foco de crisis económicas y financieras (Lewis, 1978). Esto sería un elemento para que varios países exportadores de cacao acudieran a dos soluciones: crédito bancario o crédito con las metrópolis en el caso de las colonias, e incrementar su nivel de reservas de oro pues, como mencionan Bordo y Meissner (2007), tener una posición amplia de reservas de oro reducía el peligro de acudir a deuda en moneda fuerte. De igual forma, la estructura fiscal se hizo más dependiente de los derechos de importación y exportación, este último era el 48,6\% en 1900 y el 46\% para 1910, debido a la alta concentración en las exportaciones totales de algunas de las regiones productoras: en Ghana representaba el 44,4\%22, Ecuador el 64,1\%, Venezuela el 21,4\%, mientras en Brasil fue del 2,9\% para los años previos a la Gran Guerra (Bulmer-Thomas, 1994; Glade, 1994; Leff, 1973).

Las exportaciones basadas en los cultivos condujeron a que el crecimiento económico se redujera fácilmente ante la caída de los precios y no fueran sostenibles en el largo plazo, dado por la reducción de la competitividad de varios de los cultivos del hemisferio occidental, siendo superados rápidamente por los cultivos en África. Adicionalmente, el consumo global de chocolate no tuvo en cuenta el origen del cacao, lo que hizo que la producción chocolatera se enfocara en tener la materia prima sin importar las características, la procedencia y los problemas que se presentaban en las regiones productoras. Se democratizó el consumo de chocolate, pero no necesariamente la producción de cacao, a pesar de los esfuerzos de economía política de los gobiernos. Ante la democratización del chocolate, la calidad y aroma del

22 Colonial Reports Annual. Gold Coast, \#859. 
cacao pasó a un segundo plano, ahora el cacao era un objeto destinado a un grupo masivo de consumidores.

\section{Conclusión}

La historiografía del consumo de chocolate durante el periodo previo a la Gran Guerra se ha analizado, habitualmente, a partir de los cambios en los hábitos alimenticios y el mejoramiento del ingreso desde una perspectiva donde se establecen relaciones causales. Sin embargo, la evidencia empírica procedente de la evaluación de archivos bancarios, financieros y diplomáticos, fuentes primarias impresas, así como de varias perspectivas históricas y la metodología usada, permiten establecer que el consumo global de chocolate debe ser visto como un sistema abierto y como una interacción de varios aspectos tan diversos como la política imperial, la política fiscal, la estrategia internacional de algunos países y la innovación, muy diferente a lo que en un momento se percibió como un resultado del mejoramiento del ingreso y del interés de participar en un mercado de lujo. La dinámica de los anteriores aspectos se puede ver reflejada en la transformación de las áreas cultivadas y el crecimiento de las importaciones de cacao por parte de los productores de chocolate. Primero, porque siendo un cultivo de largo plazo, en menos de treinta años, el mapa de producción cambió rápidamente y, en algunos casos, esta nueva producción, especialmente la africana, se vinculó al proceso de esclavismo y subesclavismo. Aunque en algunos territorios independientes como el Estado de Bahía en Brasil se presentaron situaciones que superaban la relación patrón-empleado como el control, el maltrato físico y la humillación. Segundo, porque los niveles de ingreso de la población con mayores niveles de consumo de chocolate no era ni siquiera la quinta parte del crecimiento de la producción de chocolate ni la importación de materia prima.

Ante estos cambios, algunas élites en Venezuela, Ecuador y Brasil se empezaron a retirar del negocio cacaotero por problemas económicos y, en algunos casos, por diversificación de actividades, lo que permitió la llegada al negocio del cacao de los pequeños agricultores y los esclavos libres que habían sido parte del modelo de producción desarrollado en los cultivos de Colombia. Por otro lado, los grandes cacaos o los senhores de engenho se convirtieron en parte importante de la definición de los asuntos públicos, la influencia económica de las regiones y su integración con las casas comerciales y los bancos privados que conectaban la venta de cacao con las empresas chocolateras en Europa y Estados Unidos. La red de parentesco soportada sobre el mayorazgo y la endogamia, que en algunos casos continuó hasta entrado el siglo $\mathrm{XX}$, mantuvo una dinámica en el cultivo de cacao en las regiones productoras de Brasil, Ecuador $y$, en menor medida, Venezuela, similar a las grandes haciendas en el Caribe y las nuevas siembras que se daban en África Occidental.

La política imperial les dio la posibilidad a los chocolateros de obtener mayores opciones para acceder a la materia prima, con esquemas de financiación a los gobiernos coloniales para que ampliaran su producción, regímenes tributarios especiales y el reconocimiento de las expansiones terratenientes que redujeran la dependencia de los productores, comerciantes y especuladores. El mercado mundial del cacao puso a varios imperios a buscar la forma de ganar la supremacía y se utilizaron los vehículos diplomáticos y financieros para lograr sus objetivos; el chocolate fue otro de los escenarios de la competencia imperial. Mientras en 1895 
los cacaos finos representaban el 79\% de las exportaciones mundiales, para 1912 eran el 30\%. Un resultado de la masificación y democratización del consumo de chocolate antes de la Gran Guerra, ya que se empezó a resaltar más la producción doméstica de los chocolates que la procedencia del cacao con el que se hacía. Es decir, se exaltaba el producto final y no la materia prima, y esto fue fundamental para transformar el consumo y la procedencia del cacao: se estaba comprando chocolate, pero no el cacao más fino. Finalmente, no hay una relación causal con un comportamiento lineal, sino que la dinámica de este grupo de aspectos fue un continuum que definió la transformación del consumo global de chocolate.

\section{Referencias}

\section{Fuentes primarias}

\section{Archivos:}

Baring Brothers \& Co., House Correspondence, The Spanish and Portuguese Correspondence. The Baring Archive.

Chicago Board of Trade Records (CBOT), Organizational Records, Annual Reports. University of Illinois at Chicago Library.

Parliamentary Debates (TPD). First and Fourth Series 1803-1908. British Library.

WR Grace \& Co. Collection (WRGC), Letterbooks WR Grace Private. Rare Book and Manuscript Library, Columbia University Library.

\section{Documentación primaria impresa:}

Bulletin of the Imperial Institute (Great Britain: Commonwealth Institute, 1906).

Bulletin of the Imperial Institute (Great Britain: Commonwealth Institute, 1908).

Colonial Reports Annual. Gold Coast. Varios años.

Colonial Reports Annual. Nigeria. Varios años.

Colonial Reports Annual. Southern Nigeria. Varios años.

Estatutos de la Compañía del Caquetá. Fundada en septiembre de 1887. Ibagué: Imprenta del Departamento.

Informe del Presidente del Estado de Santander a la Asamblea Lejislativa en sus sesiones de 1873 (1873). Socorro: Imprenta del Estado.

\section{Entrevistas:}

Pathmanathan Umaharan. En discusión con el autor. 6 de noviembre de 2015.

Xavier Elizarde Romero. En discusión con el autor. 9 de noviembre de 2012. 


\section{Fuentes secundarias}

Barker, W. H. (1925). Trade Communications in Tropical Africa. Economic Geography, 1(3), 315-336.

Blanshard, P. (1947). Democracy and Empire in the Caribbean. New York: The Macmillan Company.

Bordo, M., y Meissner, C. (2007). Financial Crises, 1880-1913: The Role of Foreign Currency Debt. En S. Edwards, G. Esquivel, y G. Márquez (Edits.), The Decline of Latin American Economies. Growth, Institutions and Crises (pp. 139-194). Chicago: The University of Chicago Press.

Bourdieu, P. (1984). Distinction: A Social Critique of the Judgment of Taste. Cambridge, MA: Harvard University Press.

Brand, R. R. (1972). The Role of Cocoa in the Growth and Spatial Organization of Accra (Ghana) Prior to 1921. African Studies Review, 15(2), 271-282.

Bulmer-Thomas, V. (1994). The Economic History of Latin America since Independence. Cambridge: Cambridge University Press.

Bushnell, D. (1993). The Making of Modern Colombia: A Nation of Itself. Berkeley, CA: University of California Press.

Cadbury, D. (2010). Chocolate Wars. London: Harper Press.

Callcott, W. (1942). The Caribbean Policy of the United States, 1890-1920. Baltimore: The Johns Hopkins Press.

Chiriloga, M. (1980). Jornaleros y Grandes Propietarios en 135 años de exportación. Quito: Consejo Provincial de Pichincha/Centro de Investigación y Estudios Socioeconómicos de Ecuador.

Clarence-Smith, W. (1990). The Hidden Costs of Labour on the Cocoa Plantations of São Tomé and Príncipe, 1875-1914. Portuguese Studies, 6, 152-172.

Clarence-Smith, W. (1994). African and European Cocoa Producers on Fernando Póo, 1880s to 1910s. The Journal of African History, 35(2), 179-199.

Clarence-Smith, W. (1995). Cocoa Plantations in the Third World, 1870s-1914. En J. Harriss, J. Hunter, \& C. Lewis (Edits.), The New institutional Economics and Third World Development (pp. 157-171). London: Routledge.

Clarence-Smith, W. (2000). Cocoa and Chocolate, 1765-1914. London: Routledge.

Clarence-Smith, W. (2016). Chocolate Consumption from the Sixteenth Century to the Great Chocolate Boom. En M. Squicciarini , y J. Swinnen (Edits.), The Economics of Chocolate (pp. 43-70). Oxford: Oxford University Press.

Clarence-Smith, W., y Ruf, F. (1996). Cocoa Pionner Fronts: The Historical Determinants. En W. Clarence-Smith (Ed.), Cocoa Pioneer Fronts since 1800 (pp. 1-22). London: MacMillan Press.

Close, F. A. (1959). High Finance in Cocoa. The Analysts Journal, 15(2), 75-80.

Coe, S., y Coe, M. (2007). The True History of Chocolate. New York: Thames \& Hudson.

Crabtree, W. A. (1914). German Colonies in Africa. Journal of the Royal African Society, 14(53), $1-14$. 
Dand, R. (2011). The International Cocoa Trade. Oxford: Woodhead Publishing.

D'Antonio, M. (2006). Hershey. New York, NY: Simon \& Schuster.

De Vries, J. (1994). The Industrial Revolution and the Industrious Revolution. The Journal of Economic History, 54(2), 249-270.

Del Valle Pavón, G. (2010). Comercialización del cacao de Guayaquil por los mercaderes del Consulado de México en la segunda mitad del siglo XVIII. Mexican Studies/Estudios Mexicanos, 26(2), 181-206.

Ferry, R. (1980). Cacao and Kindred: Transformations of Economy and Society in Colonial Caracas. PhD Dissertation: University of Minnesota.

Fitzgerald, R. (2000). Markets, Management, and Merger: John Mackintosh \& Sons, 1890-1969. Business History Review, 74(4), 555-610.

Fraser, W. H. (1981). The Coming of the Mass Market, 1850-1914. London: MacMillan Press.

Fromm, I. (2016). From Small Chocolatiers to Multinationals to Sustainable Sourcing: A Historical Review. En M. Squicciarini , \& J. Swinnen (Eds.), The Economics of Chocolate (pp. 71-87). Oxford: Oxford University Press.

Garrone, M., Pieters, H., y Swinnen, J. (2016). From Pralines to Multinationals: the Economic History of Belgian Chocolates. En M. Squicciarini, y J. Swinnen (Eds.), The Economics of Chocolate (pp. 88-118). Oxford: Oxford University Press.

Girault, A. (1916). The Colonial Tariff Policy of France. Oxford: Clarendon Press.

Glade, W. (1994). América Latina y la economía internacional, 1870-1914. En T. Halperin Donghi, W. Glade, R. Thorp, A. Bauer, M. Moreno, C. Lewis, y V. Bulmer-Thomas, Historia económica de América Latina. Desde la Independencia a nuestros días (J. Beltrán, Trad., pp. 49-97). Barcelona: Editorial Crítica.

Greaves, I. (1954). The Character of British Colonial Trade. Journal of Political Economy, 62(1), $1-11$.

Green, R. H., y Hymer, S. H. (1966). Cocoa in the Gold Coast: A Study in the Relations between African Farmers and Agricultural Experts. The Journal of Economic History, 26(3), 299-319.

Greenhill, R. (1996). A Cocoa Pioneer Front, 1890-1914: Planters, Merchants and Government Policy in Bahia. En W. Clarence-Smith (Ed.), Cocoa Pioneer Fronts since 1800. London: Macmillan Press.

Guerrero, A. (1980). Los oligarcas del cacao: ensayo sobre la acumulación originaria en el Ecuador, 1890-1910. Quito: El Conejo.

Guex, S. (1998). The Development of Swiss Trading Companies in the Twentieth Century. En G. Jones (Ed.), The Multinational Traders (pp. 150-172). London: Routledge.

Harwich, N. (1996). The Eastern Venezuela Pioneer Front, 1830s-1930s: The Role of the Corsican Trade Network. En W. Clarence-Smith (Ed.), Cocoa Pioneer Fronts since 1800 (pp. 23-44). London: Macmillan Press. 
Henderson, P. (1997). Cocoa, Finance and the State in Ecuador, 1895-1925. Bulletin of Latin American Research, 16(2), 169-186.

Henderson, W. O. (1945). British Economic Activity in the German Colonies, 1884-191. The Economic History Review, 15(1), 56-66.

Hernández, J. (2008). El fruto prohibido. El cacao de Guayaquil y el mercado Novohispano, siglos XVI-XVIII. Estudios de Historia Novohispana (39), 43-79.

Kennedy, P. (1987). The Rise and Fall of the Great Powers. New York: Vintage Books.

Knapp, A. (1920). Cocoa and Chocolate: Their History for Plantation to Consumer. London: Chapman and Hall.

Krug, C., y Quartey-Papafio, E. (1965). Análisis de la situación Cacahuera mundial. Roma: Organización de las Naciones Unidas para la Agricultura y la Alimentación.

Leff, N. H. (1973). Tropical Trade and Development in the Nineteenth Century: The Brazilian Experience. Journal of Political Economy, 81(3), 678-696.

Lewis, W. A. (1978). Growth and Fluctations, 1870-1913. London: George Allen \& Unwin Ltd.

Maiguashca, J. (1996). Ecuadorian Cocoa Production and Trade, 1840-1925. En W. Clarence-Smith (Ed.), Cocoa pioneer fronts since 1800 (pp. 65-84). London: Macmillan Press.

Martínez, A. (2000, Octubre). El chocolate, bebida sin fronteras en el siglo XIX. Revista Credencial Historia, 130.

Marzahl, P. (1978). Town in the Empire: Government, Politics and Society in Seventeenth Century Popayan. Austin, TX: Institute of Latin American Studies.

Meadows, D. (2008). Thinking in Systems. (D. Wright, Ed.) White River Junction, VT: Chelsea Green Publishing.

Mikell, G. (1992). Cocoa and Chaos in Ghana. Washington, D. C.: Howard University Press.

Miller , J., y Page, S. (2007). Complex Adaptative Systems. Princeton, NJ: Princeton University Press.

Mintz, S. (1985). Sweetness and Power: The Place of Sugar in Modern History. New York, NY: Penguin Books.

Miño, W. (2008). Breve historia bancaria del Ecuador. Quito: Corporación Editora Nacional.

Moss, S., y Badenoch, A. (2009). Chocolate: a Global History. London: Reaktion books.

Norton, M. (2006). Tasting Empire: Chocolate and the European Internalization of Mesoamerican Aesthetics. The American Historical Review, 111(3), 660-691.

Norton, M. (2008). Sacred Gifts, Profane Pleasures. A History of Tobacco and Chocolate in the Atlantic World. Ithaca, NY: Cornell University Press.

Pedler, F. (1974). The Lion and the Unicorn in Africa: The United Africa Company, 1787-1931. London: Heinemann. 
Phillips-Lewis, K. (1996). The Trinidad Cocoa Industry and the Struggle for Crown Land during the Nineteenth Century. En W. Clarence-Smith (Ed.), Cocoa Pioneer Fronts since 1800 (pp. 4564). London: Macmillan Press.

Pinero, E. (1987). Food of the Gods: Cacao and the Economy of the Province of Caracas, 1700-1770. PhD Dissertation: University of Connecticut.

Plummer, B. (1981). Race, Nationality, and Trade in the Caribbean: The Syrians in Haiti, 19031934. The International History Review, 3(4), 517-539.

Reyes, R. (1902). A través de la América del Sur: exploraciones de los Hermanos Reyes. Barcelona: Ramón de S. N. Araluce Editor.

Ross, C. (2014). The Plantation Paradigm: Colonial Agronomy, African Farmers, and the Global Cocoa Boom, 1870s-1940s. Journal of Global History, 9(1), 49-71.

Sametband, M. (1994). Entre el orden y el caos. La complejidad. Buenos Aires:: Fondo de Cultura Económica.

Satre, L. J. (2005). Chocolate on Trial: Slavery, Politics, and the Ethics of Business. Athens, OH: Ohio University Press.

Southall, R. J. (1978). Farmers, Traders and Brokers in the Gold Coast Cocoa Economy. Canadian Journal of African Studies, 12(2), 185-211.

Stern, F. (1977). Gold and Iron. Bismarck, Bleichröder and the Building of the German Empire. New York: Alfred Knopf.

Tamaro, D. (1988). A New World Plantation Region in Colonial Venezuela: Eighteenth Century Cacao Cultivation in the Tuy Valley and Barlovento. PhD Dissertation: Boston University.

Tosta Filho, I. (1957). Cocoa Economy in Brazil. Rio de Janeiro: FAO and Bank of Brazil.

Tudhope, W. (1909). The Development of the Cocoa Industry in the Gold Coast and Ashanti. Journal of the Royal African Society, 9(33), 34-45.

Volkenstein, M. (1986). Entropy and Information. (A. Shenitzer, y R. Burns, Trans.) Basel: Birkhäuser Verlag AG.

Weinman, L. J. (1970). Ecuador and Cacao:Domestic responses to the boom-collapse monoexport cycle. PhD Dissertation: University of California, Los Angeles.

Weiss, J. S. (1970). A Spectral Analysis of World Cocoa Prices. American Journal of Agricultural Economics, 52(1), 122-126.

Weymar, F. H. (1968). The Dynamics of the World Cocoa Market. Cambridge, MA: MIT Press.

Wheatley, M. (1992). Leadership and the New Science. San Francisco: Berrett-Koehler Publishers.

Wolf, E. (1982). Europe and the People without History. Berkeley, CA: University of California Press. 